\title{
How Efficient are Coronal Mass Ejections at Accelerating Solar Energetic Particles?
}

\author{
R. A. Mewaldt', C. M. S. Cohen ${ }^{\mathrm{a}}$, J. Giacalone ${ }^{\mathrm{b}}$, G. M. Mason', \\ E. E. Chollet ${ }^{b}$, M. I. Desai ${ }^{\text {, }}$ D. K. Haggerty ${ }^{\mathrm{c}}$, M. D. Looper ${ }^{\mathrm{e}}$, \\ R. S. Selesnick ${ }^{\mathrm{e}}$ and A. Vourlidas ${ }^{\mathrm{f}}$ \\ ${ }^{a}$ California Institute of Technology, Mail Code 220-47, Pasadena, CA 91125 \\ ${ }^{b}$ University of Arizona, Tucson, AZ 85721 \\ ${ }^{c}$ Johns Hopkins Applied Physics Laboratory, MS MP3-E128, Laurel, MD 20723 \\ ${ }^{d}$ Southwest Research Institute, San Antonio, TX 78238 \\ 'Aerospace Corporation, Los Angeles, CA 90009

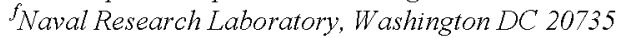

\begin{abstract}
The largest solar energetic particle (SEP) events are thought to be due to particle acceleration at a shock driven by a fast coronal mass ejection (CME). We investigate the efficiency of this process by comparing the total energy content of energetic particles with the kinetic energy of the associated CMEs. The energy content of 23 large SEP events from 1998 through 2003 is estimated based on data from ACE, GOES, and SAMPEX, and interpreted using the results of particle transport simulations and inferred longitude distributions. CME data for these events are obtained from SOHO. When compared to the estimated kinetic energy of the associated coronal mass ejections (CMEs), it is found that large SEP events can extract $\sim 10 \%$ or more of the CME kinetic energy. The largest SEP events appear to require massive, very energetic CMEs.
\end{abstract}

Keywords: : solar energetic particles, CMEs, particle acceleration.

PACS: $95.50 . \mathrm{Vg}, 96.50 . \mathrm{Pw}, 96.50 \mathrm{sh}, 96.50 . \mathrm{sb}$. 96.60.ph

\section{INTRODUCTION}

The largest solar energetic particle (SEP) events occur in association with violent eruptions on the Sun in which coronal mass ejections (CMEs) containing more than $10^{15} \mathrm{~g}$ of material are accelerated to speeds of $\sim 1000-2500 \mathrm{~km} / \mathrm{s}$. Most of the SEPs observed in interplanetary space in these large events are thought to be the result of acceleration at a shock driven by the CME, although particles are also accelerated by other process in association with the accompanying flare. With the launch of ACE in 1997 it became possible to measure the energy spectra of the abundant species of solar energetic particles (SEPs) over $>3$ orders of magnitude in energy, from $\sim 50 \mathrm{keV} / \mathrm{nuc}$ to $\sim 100 \mathrm{MeV} /$ nuc (see, e.g., $[1,2]$ ). Among other studies, the ACE measurements (supplemented by other data for high-energy $\mathrm{H}$ and $\mathrm{He}$ ) enable improved assessments of the energy content of particles accelerated in SEP events for comparison with other contributions to the energy budget of large solar eruptive events. Emslie et al. [3,4] tabulated the energy budget for two large solar events, one of which (21 April 2002) resulted in a large SEP event at $1 \mathrm{AU}$.

\footnotetext{
CP1039, Particle Acceleration and Transport in the Heliosphere and Beyond- $7^{\text {th }}$ Annual Astrophysics Conference edited by G. Li, Q. Hu, O. Verkhoglyadova, G. P. Zank, R. P. Lin, and J. Luhmann (O) 2008 American Institute of Physics 978-0-7354-0566-0/08/\$23.00
} 
One surprising result of the April 21 comparison was that the estimated SEP energy content was $\sim 15 \%$ of the estimated kinetic energy of the associated coronal mass ejection (CME). Assuming that the bulk of the observed SEPs in these large events are the result of shock acceleration driven by the CME [5], this suggests that CMEdriven shock acceleration can be a rather efficient process. Mewaldt et al. [1] extended this comparison to five events from the 2003 "Halloween" period and found apparent acceleration efficiencies ranging from $\sim 0.4 \%$ to $\sim 20 \%$. A later study [6] extended this comparison to 17 large SEP events, with similar results. In this paper we compare SEP and CME kinetic energies in 23 events, including for the first time a correction for the adiabatic energy loss that SEPs suffer as they scatter off fluctuations in the interplanetary magnetic field.

\section{OBSERVATIONS AND KINETIC ENERGY ESTIMATES}

The SEP observations reported here were made by the SIS, ULEIS, and EPAM instruments on ACE, the PET instrument on SAMPEX, and the EPS sensors on NOAA's GOES-8 and GOES-11 satellites. Figure 1 shows energy spectra for $\mathrm{H}, \mathrm{He}$, and $\mathrm{O}$ for the 2 November 2003 event along with fits using the double-power law spectral form of Band et al. [7]. This spectral shape provides excellent fits to the majority of the observed spectra [1]; the Ellison-Ramaty [8] spectral shape (power law with an exponential cutoff) was fit to the remaining spectra. As part of another study, we measured and fit proton energy spectra for the 50 largest SEP events of solar cycle 23 (ordered by the fluence of $>30 \mathrm{MeV}$ protons measured by GOES).

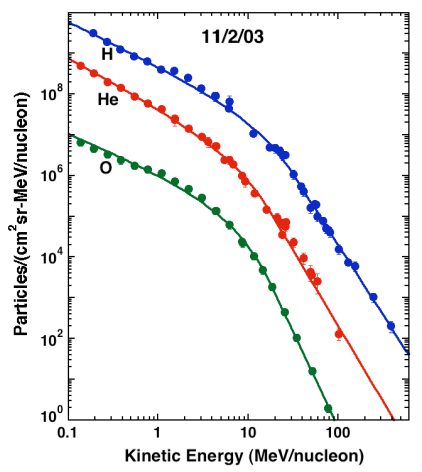

Figure 1: H, He, and O fluence spectra for the SEP event of 2 November 2003 [1], fit with the double power-law formula of Band et al. [7].

The CME observations were made by the LASCO instrument on SOHO. The mass of a CME can be estimated from its total brightness and combining this with the CME velocity leads to an estimate of the kinetic energy $[9,10]$. Gopalswamy et al. summarize mass and velocity estimates for CMEs observed during 1996-2002 [11] and during the Halloween 2003 period [12]. We include those top 50 SEP events for which we found published CME mass and velocity measurements, eliminating events where contributions from multiple CMEs could not be separated. 
The average properties of CMEs observed during 1996-2003 were tabulated by Gopalswamy et al. [13], who found mean values of $\sim 7 \times 10^{14} \mathrm{~g}$ for the mass, 483 $\mathrm{km} / \mathrm{sec}$ for the velocity, and $\sim 5 \times 10^{29}$ ergs for the kinetic energy. In Figure 2 we plot CME quantities for 1996-2003 CMEs and for those top $50 \mathrm{SEP}$ events (>30 MeV proton fluences of $>4 \times 10 \% \mathrm{~cm}^{2}$ ) with CME mass and velocity data. Note that the largest SEP events do not result from average CMEs - only a small percentage of CMEs, those that are most massive and most energetic, produce large SEP events.
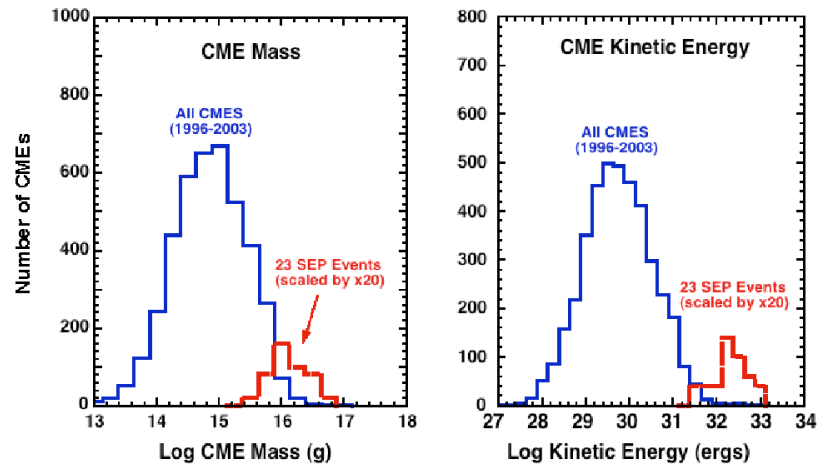

Figure 2: Histograms of the log of the mass (left) and kinetic energy (right) distributions of all CMEs [12,13] and of those CMEs (when available) associated with the 23 of the largest SEP events of solar cycle 23. The histogram for the 23 SEP events is scaled up by a factor of 20

The energy spectra measured at $1 \mathrm{AU}$ can be used to estimate the SEP energy/ $\mathrm{cm}^{2}$ escaping into the outer heliosphere. We wish to relate this to the corresponding energy required to accelerate the particles that traversed this $1-\mathrm{cm}^{2}$ area. To do this we need to correct for the fact that a given particle may have crossed 1 AU a number of times, providing several opportunities to be observed.

To estimate the number of 1-AU crossings as a function of energy and species we used simulations by J. Giacalone and E. Chollet (see also [14]). In these simulations $500,000 \mathrm{H}$ and $\mathrm{He}$ ions of several energies were released isotropically at $0.1 \mathrm{AU}$ and followed as they moved through the heliosphere with an scattering mean free path of $0.1(\mathrm{M} / \mathrm{Q})\left(\mathrm{R} / \mathrm{R}_{\mathrm{o}}\right) \mathrm{AU}$, where $\mathrm{M}$ is the particle mass number, $\mathrm{Q}$ its mean charge state, $\mathrm{R}$ the rigidity, and $R_{0}=43.3 \mathrm{MV}$. The interplanetary magnetic field was taken to be a Parker spiral with a solar wind speed of $400 \mathrm{~km} / \mathrm{s}$. Pitch angle diffusion, convection, and adiabatic energy-loss were accounted for as the individual particles moved. Each time a particle crossed $1 \mathrm{AU}$ it was counted and its remaining kinetic energy recorded. Figure 3 shows distributions of the number of 1-AU-crossings made by $\mathrm{H}$ and $\mathrm{He}$ ions that started out with $1 \mathrm{MeV}$. Also shown are the distributions of the remaining kinetic energy. The number of crossings $\left(\mathrm{N}_{\mathrm{c}}\right)$ is somewhat greater for $\mathrm{H}$ than for $\mathrm{He}$ because protons scatter more. The fraction of the original energy $(F)$ is therefore somewhat smaller for protons.

The source energy S needed to account for the SEP energy escaping into the outer heliosphere can be evaluated using the following integral: 


$$
\mathrm{S}=\pi \int(\mathrm{d} J / \mathrm{dE}) \mathrm{E} /\left[\mathrm{F}\left(\mathrm{E}_{\mathrm{s}}\right) \mathrm{N}_{\mathrm{c}}\left(\mathrm{E}_{\mathrm{S}}\right)\right] \mathrm{dE}
$$

where $\mathrm{dJ} / \mathrm{dE}$ is a fit to the observed spectrum, and $\mathrm{F}\left(\mathrm{E}_{\mathrm{s}}\right)$ and $\mathrm{N}_{\mathrm{c}}\left(\mathrm{E}_{\mathrm{s}}\right)$ are evaluated at the source energy $E_{s}=E / F\left(E_{s}\right)$. This approach makes the approximation that all particles observed at a given energy, E, are assumed to have lost the same fraction of their original energy (e.g., all $565 \mathrm{keV}$ protons were assumed to have started at $1 \mathrm{MeV}$; see Figure 3). The limits of the integration were from $10 \mathrm{keV} / \mathrm{nuc}$ to $1000 \mathrm{MeV} / \mathrm{nuc}$, which required some extrapolation of the fits. However, in most cases the contribution to the integral that relies on extrapolation was $<10 \%$.
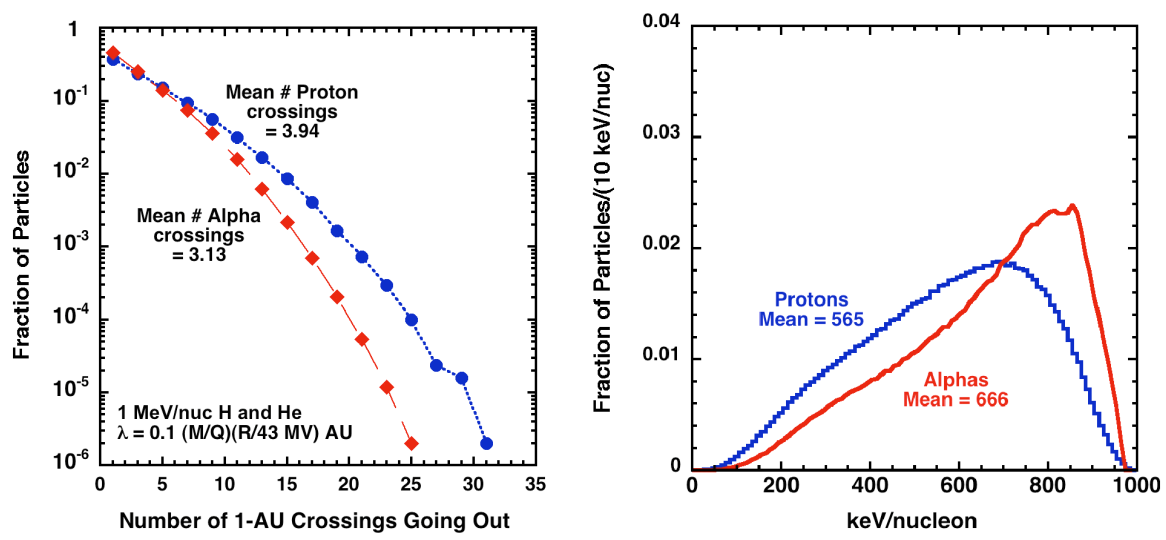

FIGURE 3. Simulations for $500,0001-\mathrm{MeV}$ protons released at $0.1 \mathrm{AU}$. The scattering mean free path was $0.1(\mathrm{M} / \mathrm{Q})(\mathrm{R} / \mathrm{Ro}) \mathrm{AU}$ where $\mathrm{R}$ is rigidity and $\mathrm{Ro}=43.3 \mathrm{MV}$. Shown on the left is the distribution of the number of times $1-\mathrm{MeV} / \mathrm{nuc} \mathrm{H}$ and He crossed $1 \mathrm{AU}$ (going out) in the 5 days following release. The corrections for multiple crossing are somewhat greater than used in $[1,3,6]$. Shown on the right is the distribution of the ion kinetic energy each time they crossed $1 \mathrm{AU}$. Protons lose a greater fraction of their energy because they have a shorter mean free path.

To estimate the total energy input requires assumptions about the longitudinal and latitudinal distribution of SEPs. Following earlier work $[3,1,6]$ we assume that the largest near-Earth events originate at central meridian (based on NOAA and ACE data), and that the near-Earth fluence for events that originate away from the central meridian falls off exponentially with e-folding values of $25^{\circ}$ for eastern events and $45^{\circ}$ for events originating to the west. The latitudinal e-folding value was taken to be $35^{\circ}$. With these corrections and Eqn. 1 we can relate the fluence observed at a given location to the total number of accelerated particles and their energy content.

It is difficult to evaluate the uncertainties in the corrections described above, but as an estimate we assume that the uncertainty in a correction of size $\mathrm{x}$ is equal to $\sqrt{x}_{\mathrm{x}}$. The uncertainties for the location and crossings/energy-loss corrections are added in quadrature. Simulations using other MFP values indicate that these corrections are not very sensitive to the assumed MFP because the multiple-crossing and energy-loss corrections tend to cancel (more scattering increases $\mathrm{N}_{\mathrm{c}}$ but decreases $\mathrm{F}$ ). There are also uncertainties in the CME kinetic energy estimates. Gopalswamy [15] estimates a factor of $\sim 2$ uncertainty for limb CMEs, with larger uncertainties for halo CMEs. 
For six events [1,3] we have measured the spectra of $\mathrm{H}, \mathrm{He}$, and abundant heavier ions ranging from $\mathrm{C}$ to $\mathrm{Ni}$, as well as electrons from $35 \mathrm{keV}$ to $8 \mathrm{MeV}$. Using these results we found that $\mathrm{H}$ accounted for $69 \%$ to $82 \%$ of the total SEP energy; $\mathrm{He}$ accounted for $10 \%$ to $19 \%$; heavy ions from $\mathrm{C}$ to $\mathrm{Ni}$ accounted for $3 \%$ to $10 \%$; and electrons from $1 \%$ to $11 \%$. For the remaining events we have corrected the $\mathrm{H}$ spectra, and assumed that protons accounted for $75 \pm 7 \%$ of the total energy.

Before comparing SEP and CME kinetic energies we need to consider that CMEs will not form a shock unless they are traveling substantially faster than the ambient solar wind. To form a shock it is required that $\mathrm{v}_{\mathrm{cme}}>\mathrm{v}_{\mathrm{sw}}+\mathrm{v}_{\text {fast, }}$, where $\mathrm{v}_{\text {fast }} \approx\left(\mathrm{v}_{\mathrm{A}}{ }^{2}+\right.$ $\left.c_{s}^{2}\right)^{1 / 2}$. Here $V_{A}$ is the Alfven speed and $c_{s}$ is the sound speed (see e.g., [16]). However, the Alfven velocity is thought to be highly variable in the corona and also decreases with distance from the Sun. The particles in these large events were accelerated over a range of distances, starting close to the Sun and continuing at evergreater distances as the shock moves out. Gopalswamy et al. [17] estimate that between $\sim 3$ and $\sim 11 \mathrm{Rs}$, over an active region, a CME speed of $\sim 300-400 \mathrm{~km} / \mathrm{s}$ is needed to form a shock. To approximate the energy available for accelerating particles, we compare the SEP kinetic energy to an estimate of the CME kinetic energy in the rest frame of the solar wind, which we evaluate by subtracting $400 \mathrm{~km} / \mathrm{s}$ from all $\mathrm{CME}$ velocities before computing the $\mathrm{CME}$ kinetic energy. Then, $\mathrm{KE}_{\mathrm{CME}}=$ $(1 / 2) \mathrm{M}_{\mathrm{CME}}\left(\mathrm{V}_{\mathrm{CME}}-400 \mathrm{~km} / \mathrm{s}\right)^{2}$.
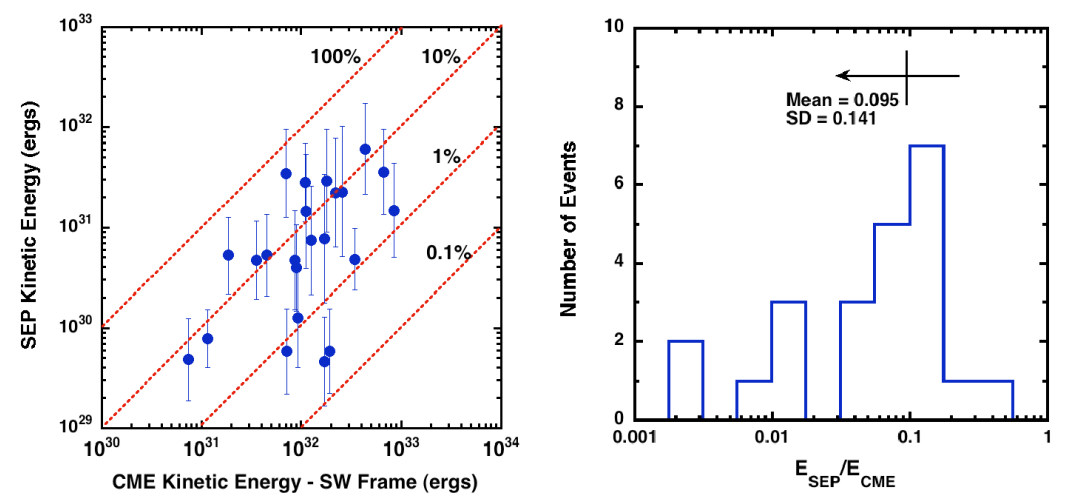

Figure 4: (Left): Total SEP kinetic energy (in the solar wind rest frame; see text) versus the kinetic energy of the corresponding CME [11,12]. SEP uncertainties are discussed in the text; the CME kinetic energy estimates are also uncertain by a factor of $\sim 2$ or more (15). (Right): Histogram of the ratio of the SEP and CME kinetic energies. The mean and standard deviation are indicated.

\section{RESULTS AND DISCUSSION}

A comparison of the resulting SEP and CME kinetic energies in the rest frame of the solar wind is shown in the left panel of Figure 4. Also shown on the right is a histogram of the acceleration efficiency, defined to be the ratio of SEP and CME kinetic energies in the rest frame of the solar wind. Although the uncertainties are large, there appear to be a number of events near the $\sim 10 \%$ efficiency line. The mean 
efficiency is $9.5 \%$ with a standard deviation of $14.1 \%$, and the median efficiency is $6.5 \%$. It is encouraging that there are no events where the SEP kinetic energy exceeds the available CME energy.

As noted above, this is the first time that adiabatic energy loss has been taken into account in obtaining the SEP energy estimates. For the largest events the energy-loss correction increased the kinetic energy estimates by $\sim 30 \%$; for small events with soft spectra the increase was somewhat greater.

While the CME acceleration efficiency estimated here may seem high, it is interesting that the acceleration of galactic cosmic rays by supernova shocks requires that an average of $\sim 15 \%$ of the energy release in a supernova explosion go into accelerating galactic cosmic rays (GCRs) [18] in order to keep the GCR energy density sustained over the $\sim 15 \mathrm{MY}$ lifetime of cosmic rays in the Galaxy [19].

Several factors apparently affect the SEP acceleration efficiency, including preconditioning by earlier CMEs [11], pre-existing turbulence [20], and the density of suprathermal seed particles [21]. In future work we hope to use this data set to isolate the conditions that contribute to making the largest SEP events of the solar cycle.

\section{SUMMARY AND FUTURE OUTLOOK}

In this study we have estimated the total kinetic energy of accelerated particles in 23 of the largest SEP events of solar cycle 23, including all of the 50 largest SEP events for which we could find published measurements of CME mass and speed. These SEP events are found to be associated with the most massive $\left(\sim 10^{16} \mathrm{~g}\right)$ and most energetic $\left(\sim 10^{32} \mathrm{ergs}\right)$ CMEs of the solar cycle. After correcting for effects of particle transport and magnetic connection, we find that the kinetic energy of the accelerated particles is, on average, $\sim 9.5 \%$ of the kinetic energy of the associated CMEs, where the CME kinetic energy was estimated in the rest frame of the solar wind. Although there are sizeable uncertainties in these comparisons, we conclude that CME-driven shock acceleration can be a very efficient process.

During the next few years we can expect significant progress in several areas. New SEP transport simulations by the University of Arizona team are being used to test the sensitivity of the multiple-crossing and energy-loss corrections over a range of transport parameters, including radial dependence. There is also an NASA-LWS sponsored effort involving LASCO investigators to obtain improved CME energy estimates, including solar cycle 23 events and estimated uncertainties.

NASA's STEREO mission, launched in October 2006, is designed to provide multi-point imaging and in situ observations of CME and SEP events [22,23,24,25]. With two STEREO points of view (and a third from SOHO) there will be a valuable cross-check on the CME mass and velocity estimates. With multiple in situ measurements of SEPs and ICME plasma, it is likely that one spacecraft will always be magnetically well-connected, and it will be possible to measure directly the longitudinal distribution of SEPs and ICME shocks and ejecta in interplanetary space.

Finally, by using an improved relationship between CME and SEP energies it should be possible to use real-time CME kinetic energy estimates (as well as connection longitude and other data) to predict the maximum eventual fluence of an SEP event within the first few hours following an eruption on the Sun [26]. 


\section{ACKNOWLEDGMENTS}

This work was supported by NASA under grants NAG5-12929, NNG04GB88B, and NNX06AC21G. We appreciate the availability of GOES data from NOAA and CME data from SOHO. We acknowledge helpful suggestions by Dave McComas. Finally, we thank the organizers for a very productive and enjoyable conference.

\section{REFERENCES}

1. R. A. Mewaldt, et al., J. Geophys. Res., 110, A09S18, doi:10.1029/2005JA011038 (2005).

2. C. M. S. Cohen et al. J. Geophys. Res., 110, A09S16, doi:10.1029/2005JA011004 (2005).

3. A. G. Emslie, et al., J. Geophys. Res., 109, A10104, doi:10.1029/2004JA010571 (2004).

4. A. G. Emslie, et al., J. Geophys. Res., 110, A11103, doi:10.1029/2005JA011305 (2004).

5. H.V. Cane, et al., J. Geophys. Res., doi:10.1029/2005JA011071 (2006).

6. R. A. Mewaldt, et al., in Connecting Sun and Heliosphere, Proc. Solar Wind $11-$ SOHO-16, ESA SP-592, pp. 67 (2005).

7. D. Band, et al., Astrophys. Journal, 413, 281, (1993).

8. D. C. Ellison, and R. Ramaty, Astrophys. Journal, 298, 400 (1985).

9. A. Vourlidas, et al., Astrophys. Journal, 534, 456 (2000).

10. A. Vourlidas, D. Buzasi, R. A. Howard and E. Esfandiari, in Solar Variability: From Core to Outer Frontiers. Ed. A. Wilson. ESA SP-506, Vol. 1. Noordwijk: ISBN 92-9092-816-6, p. 91, (2002).

11. N. Gopalswamy, et al., J. Geophys. Res., 109, A12105, doi:10.1029/2004JA10602 (2004).

12. N. Gopalswamy, et al., JGR, 110, A9, A09S15, doi:10.1029/2004JA01095813 (2005).

13. N. Golpalswamy, J. Astrophys. Astr. 27, 243 (2006).

14. G. Li, G. P. Zank, and W. K. M. Rice: J. Geophys. Res. 108, doi:10.1029/2002JA009666 (2003).

15. N. Gopalswamy, personal communication, (2008).

16. S. W. Kahler and D. V. Reames, Astrophys. J. 584, 1063 (2003).

17. N. Gopalswamy, et al., J. Geophys. Res. 106, 25261 (2001).

18. V. S. Ptuskin, Space Sci Rev. 99, 281 (2001).

19. N. E. Yanasak, Astrophys. J. 563, 768 (2001).

20. G. Li and G. P. Zank, Proc. 29th Internat. Cosmic Ray Conf., 1, 173 (2005).

21. R. A. Mewaldt, C. M. S. Cohen, and G. M. Mason, in Solar Eruptions and Energetic Particles, N. Gopalswamy, R. A. Mewaldt, and J. Torsti, eds, AGU Monograph Series, pp. 115 (2006).

22. R. A. Howard, et al., Space Science Rev. 136, 67, doi:10.1007/s1 1214-008-9341-4 (2008).

23. J. G. Luhmann, et al., Space Science Rev., 136, 117, doi:10.1007/s11214-007-9170-x (2008).

24. R. A. Mewaldt, et al., Space Science Rev., 136, 285, doi:10.1007/s11214-007-9288-x (2008).

25. Galvin, A. B., Space Science Rev., 136, 437, doi:10.1007/s11214-007-9296-X (2008).

26. R. A. Mewaldt, et al., in Turbulence and Nonlinear Processes in Astrophysical Plasmas, AIP Conference Proc. \#932, D. Shaikh and G. P. Zank, eds. p. 277 (2007). 